\title{
COMPROMISO ESTUDIANTIL EN EL DESEMPEÑO ACADÉMICO Y SUS DIFERENCIAS POR GÉNERO
}

\author{
Erika Yunuen Morales Mateos ${ }^{1}$ \\ María Arely López Garrido² \\ Laura López Díaz
}

\begin{abstract}
The purpose of this research was to identify groups of students characterized by their student commitment. There were 31 participating students belonging to careers related to information technology from a university un the southern of Mexico. For this, the authors applied the UWES-S with a series of questions related to the academic fields. The data mining technique called clustering was subsequently applied to identify the group using the WEKA tool. It is highlighted as a result that the group of women has high levels of student's commitment, vigor and absorption, compared to men, who have a high level of dedication.
\end{abstract}

Keywords: Student commintment, $\mathrm{KDD}$, data mining technique, clustering.

\section{Introducción}

En atención a las demandas nacionales e internacionales las Instituciones de Educación Superior han adaptado sus modelos educativos a nuevos esquemas administrativos, académicos y normativos con el objetivo de mejorar la calidad de los planes y programa de estudio, estos modelos están centrados en el alumno y su aprendizaje; en este, los alumnos tienen la responsabilidad y el compromiso de su propio aprendizaje mediante una participación activa, significativa $\mathrm{y}$ experimental, por lo cual, los profesores deben de estimular la motivación y participación (UJAT, 2006).

Por lo anterior el compromiso académico definido como "un estado mental positivo relacionado con el

\footnotetext{
${ }^{1}$ Profesora Investigadora en la División Académica de Informática y Sistemas, Universidad Juárez Autónoma de Tabasco. Cunduacán, Tabasco México.

${ }^{2}$ Profesora Investigadora en la División Académica de Informática y Sistemas, Universidad Juárez Autónoma de Tabasco. Cunduacán, Tabasco México.

${ }^{3}$ Profesora Investigadora en la División Académica de Informática y Sistemas, Universidad Juárez Autónoma de

Tabasco. Cunduacán, Tabasco México y en la Universidad los Ángeles en Comalcalco, Tabasco, México
} 
trabajo y caracterizado por vigor (altos niveles de energía y resistencia mental), dedicación (alta implicación laboral) y absorción (alto estado de concentración e inmersión)", es importante en el desempeño académico (Schaufeli y Bakker, 2003).

Se han realizado varias investigaciones del compromiso estudiantil y su relación con el desempeño académico, algunos de estos estudios se mencionan a continuación:

En la UNHEVAL, se realizó una investigación del compromiso escolar y su relación con el rendimiento académico, el instrumento utilizado para medir el compromiso escolar fue el cuestionario Utrech Work Engagement Scale Student o UWES-S (versión extendida de 17 items), obtuvieron resultado de manera general que el compromiso estudiantil no incide en el rendimiento, aunque si hay relación entre el compromiso escolar y el rendimiento académico en estudiantes de Enfermería y Obstetricia respectivamente(Muñoz y Morales, 2019).

Un estudio realizado en la Universidad de León para determinar el nivel de vinculación con los estudios, la muestra fue un grupo de 499 alumnos, los resultados obtenidos muestran que los alumnos que cursan la carrera elegida como primera opción presentan mayores niveles de engagement y rendimiento académico que los demás, por lo anterior concluyen la importancia de desarrollar programas de orientación vocacional (Gómez y Gundin, 2016).

En una investigación con estudiantes de Enfermería, de cuatro universidades públicas de España: Universidad de Burgos, Universidad de León, Universidad de Salamanca y Universidad de Valladolid, en el cual se describe el nivel de bornout (agotamiento, cinismo y eficacia académica) y el compromiso académico a través del engagement (absorción, dedicación y vigor), dentro de los resultados observan que las mujeres se perciben con puntuaciones más bajas en cinismo y más altas en eficacia académica, absorción y dedicación (Liébana-Presa, et al, 2018).

\section{Métodos y herramientas}

La metodología empleada en el presente estudio fue de corte cuantitativo, el cual se caracteriza por ser racional, objetivo, se basa en lo observable, en lo manipulable $\mathrm{y}$ 
verificable [9]. En este caso se analizaron los estilos de jugadores que prevalecen en los estudiantes universitarios estableciendo variables y reglas de asociación entre ellas, con la finalidad de implementar en un futuro un proceso de gamificación lo más efectivo en relación a sus preferencias en el juego. La población de este estudio se encuentra integrado por 22 estudiantes de la División Académica de Informática y Sistemas (DAIS) de la Universidad Juárez Autónoma de Tabasco (UJAT) de $1^{\circ}$ año de la carrera de Ingeniería en Sistemas Computacionales, que cursaron la asignatura Algoritmos en el verano de 2019.

\section{Modelo kdd}

El modelo empleado en la presente investigación es el descubrimiento de conocimiento en base de datos (Knowledge Discovery in Databases, KDD). Este modelo es un proceso, interactivo e iterativo, algunas veces es dependiendo de las salidas es necesario volver a pasos anteriores. El modelo KDD se divide en 5 etapas (ver. Fig 1) las cuales se describen a continuación (Hernández, Ramírez, Ferri, 2004):
- Integración $\mathrm{y}$ recopilación de datos: Se recopilan los datos iniciales identificando las fuentes de información que pueden ser útiles, estos datos generalmente se encuentran en fuentes de datos internas o externas, todas laas fuentes de información se transforman y se pasan a un mismo formato.

- Selección, limpieza y transformación: Se revisan los datos, de manera de que se identifiquen si hay datos faltantes o duplicados para darle el tratamiento adecuado, se describen estadísticamente los datos y se seleccionan los datos cuyas variables son de relevancia para la investigación.

- Minería de datos: En esta fase se selecciona el método, modelo y algoritmo de la minería de datos que se va a emplear y se aplican a los datos para obtener conocimiento.

\section{- Evaluación}

interpretación: Se evalúan los patrones en cuanto a si son precisos, compresibles e interesantes, lo que puede implicar volver a las fases anteriores.

- Difusión: Se utiliza el nuevo modelo, se distribuye y comunica a los posibles usuarios. 


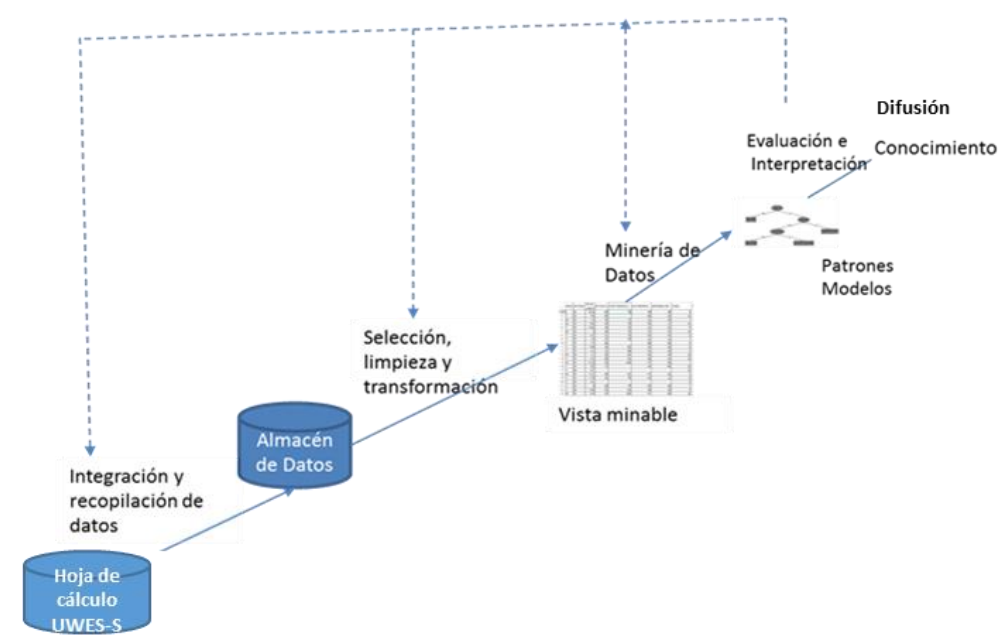

Fig. 1. Proceso de Descubrimiento del conocimiento del KDD.

Fuente: Fayyad, Piatetsky-Shapiro, Smyth, 1996..

\section{Minería de datos}

La minería de datos cosiste en un conjunto de técnicas para el descubrimiento de conocimiento en grandes volúmenes de datos, analiza comportamientos, patrones, tendencias, asociaciones (Pérez, 2014).

El principal objetivo de la minería de datos es que a través del análisis de grandes cantidades de datos se genere conocimiento. Los retos a los que se enfrenta la minería de datos es trabajar con grandes cantidades de datos que generalmente provienen de sistemas de adecuada para obtener conocimiento novedoso y útil (Hernández et al., 2004).
Las tareas de la minería de datos pueden ser descriptivas o predictivas. Son tareas predictivas: la clasificación y la regresión y son descriptivas el agrupamiento, las reglas de asociación secuencias y las correlaciones.

\section{Agrupamiento (clustering)}

$\mathrm{El}$ agrupamiento (clustering) es una tarea descriptiva, que consiste en identificar grupos de objetos lo más diferentes entre grupos y lo más similares dentro de los grupos. (Pérez, 2014).

Una de las técnicas para el agrupamiento es el algoritmo $\mathrm{K}$ medias, este es un método basado en la vecindad, 
se parte de un número determinado de prototipos y de un conjunto de ejemplos a agrupar, sin etiquetar. $\mathrm{K}$ medias sitúa a los prototipos o centros en el espacio, y de esta manera incluye los datos pertenecientes al mismo prototipo con características $\quad$ similares. $\quad$ El procedimiento de $\mathrm{K}$ medias es el siguiente [Hernández et al., 2004):

- Se calcula, para cada ejemplo xk, el prototipo más próximo Ag y se incluye en la lista de ejemplos de dicho prototipo.

- Después de haber colocado todos los ejemplos, cada prototipo Ak tendrá un conjunto de datos a los que representa.

- $\quad$ Se desplaza el prototipo hacia el centro de masas del conjunto que representa.

- $\quad$ Se repite el procedimiento hasta que ya no se desplazan los prototipos.

\section{Software weka}

El software de minería de datos WEKA (Waikato Environment for Knowledge Analysis) es una colección de algoritmos de aprendizaje automático para tareas de min ería de datos. Está compuesto por paquetes de código abierto emitido bajo la Licencia Pública General GNU, está desarrollado en Java, incluye diferentes técnicas de preprocesado, clasificación, agrupamiento, asociación y visualización. Incluye una interfaz gráfica que facilita su uso; de código abierto (Weka, 2019). Este software fue el seleccionado para aplicar la técnica de agrupamiento.

\section{Desarrollo del análisis de datos para la} obtención de agrupaciones del compromiso estudiantil

El conjunto de datos de esta investigación se encuentra conformado por 31 registros de estudiantes, un total de 12 mujeres y 19 hombres, de carreras relacionadas con tecnologías de la información de una universidad del sur de México. Luego de obtener los resultados del instrumento UWES-S, se analizaron los datos generando sus estadísticos descriptivos, entre ellos: el valor mínimo, máximo, el promedio, la moda y la mediana.

La Tabla 1 y la Tabla 2 muestran los resultados obtenidos por la población estudiada para hombres $\mathrm{y}$ mujeres respectivamente. Se puede observar en la Tabla 1 que el 
compromiso estudiantil en hombres, tiene un valor promedio de 4.5 considerado alto en la escala de medida, y valores mínimo de 2.7 y máximo de 5.8 respectivamente; en cuanto a su promedio académico la media es de 8.2, para las dimensiones que comprenden el compromiso estudiantil, el valor promedio puntuado como más alto es el de dedicación con 5.3, seguido de la absorción con 4.3, y finalmente el vigor con 4.1; así mismo se presentan los valores correspondientes a la moda y mediana para cada una de las variables.

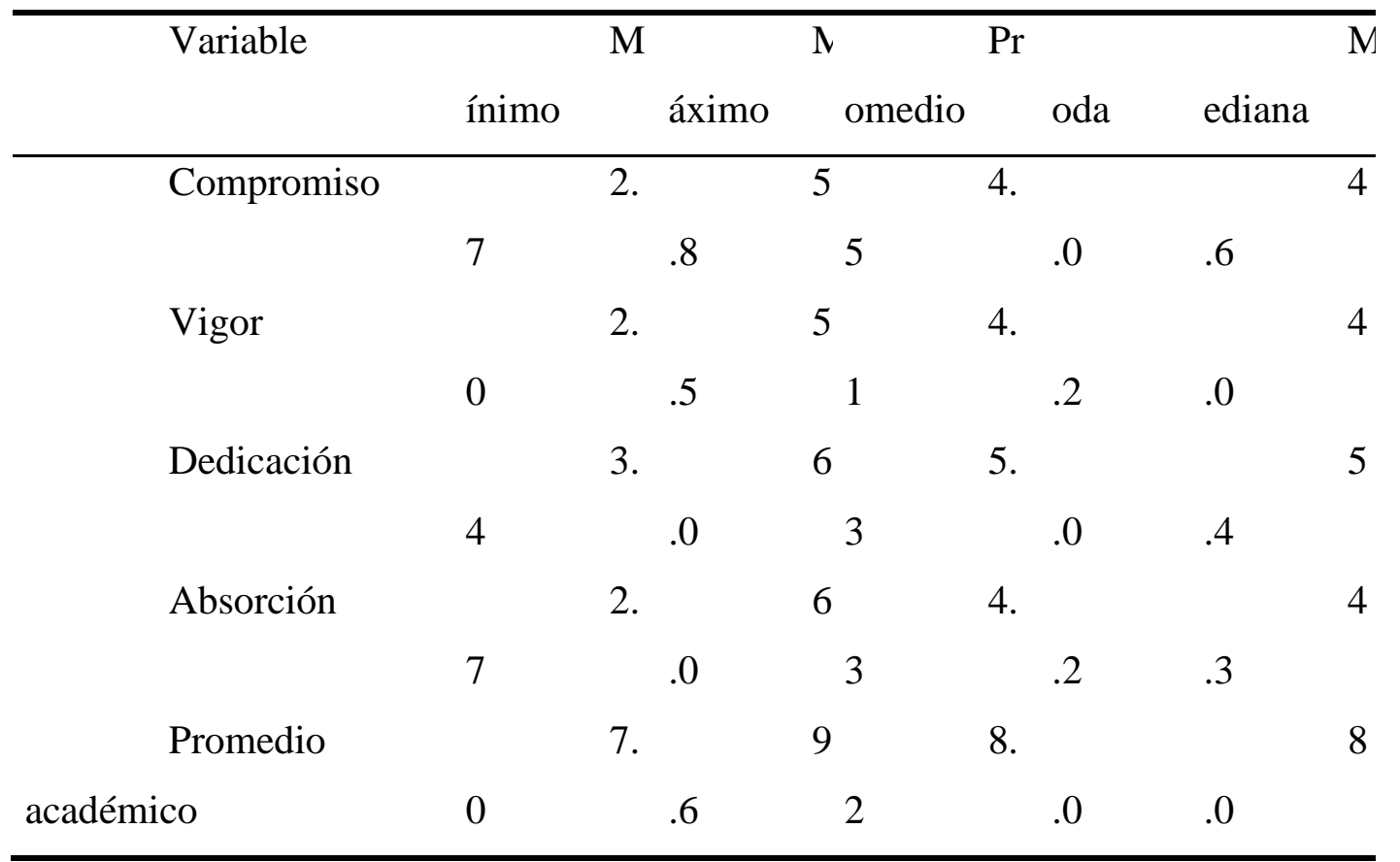

Tabla 1. Estadísticos descriptivos del conjunto de datos: hombres. Elaboración propia.

En la Tabla 2 se muestra que el compromiso estudiantil en mujeres tiene un valor promedio de 4.8 considerado alto en la escala de medida, y valores mínimo de 3.5 y máximo de 5.9 respectivamente; superando a los valores obtenidos en por los estudiantes hombres; en cuanto a su promedio académico la media es de 8.4, para las dimensiones que comprenden el compromiso estudiantil, el valor promedio puntuado como más alto es el de dedicación con 5.2, seguido de la absorción con 4.5, y finalmente el vigor 
con 4.6; así mismo se presentan los valores correspondientes a la moda y mediana para cada una de las variables. En comparación entre hombres y mujeres, se observa que las mujeres presentan valores promedios más altos en compromiso, vigor, absorción y adicionalmente un promedio más alto.

\begin{tabular}{|c|c|c|c|c|c|c|c|c|}
\hline \multirow[b]{2}{*}{ ble } & \multicolumn{2}{|l|}{ Varia } & \multirow{2}{*}{$\begin{array}{l}\mathrm{N} . \\
\text { áximo }\end{array}$} & \multirow{2}{*}{$\mathrm{M}$} & \multirow{2}{*}{ romedio } & $\bar{P}$ & \multirow{2}{*}{$\begin{array}{l}\mathrm{N} \\
\text { diana }\end{array}$} & \multirow[t]{2}{*}{$\mathrm{Me}$} \\
\hline & & ínimo & & & & oda & & \\
\hline & Comp & & 3 & 5. & & 4. & 4 & 4.9 \\
\hline \multirow[t]{4}{*}{ romiso } & & .5 & 9 & & 8 & .9 & & \\
\hline & Vigor & & 3 & 6. & & 4. & 4 & 4.5 \\
\hline & & .5 & 0 & & 6 & .3 & & \\
\hline & Dedic & & 3 & 6. & & 5. & 5 & 5.4 \\
\hline \multirow[t]{2}{*}{ ación } & & .4 & 0 & & 2 & .4 & & \\
\hline & Absor & & 3 & 5. & & 4. & 4 & 4.7 \\
\hline \multirow[t]{2}{*}{ ción } & & .5 & 7 & & 5 & .8 & & \\
\hline & Prome & & 7 & 9. & & 8. & 8 & 8.4 \\
\hline dio & & .9 & 7 & & 4 & .0 & & \\
\hline
\end{tabular}

Tabla 1. Estadísticos descriptivos del conjunto de datos: mujeres. Elaboración propia.

\section{Fases del kdd}

$$
\text { Para desarrollar la }
$$

corresponde a la extensión cvs, delimitado por comas.

clusterización se empleó el proceso del KDD, el cual se describe a continuación:

\section{Integración y recopilación de datos}

Se recopilaron y organizaron los datos obtenidos con el instrumento UWES-S y las preguntas del ámbito académico en un formato legible para ser usar en el software Weka, el cual

\section{Selección limpieza y transformación}

En esta etapa del estudio se revisan los datos, seleccionando aquellos con características que son de relevancia para la investigación.

\section{Evaluación e interpretación}


Para la validación de esta técnica dada la cantidad de registros en el conjunto de datos, se aplicó la opción use training set de Weka. Esta opción entrena el método con todos los datos disponibles y después se lleva a cabo la evaluación sobre los mismos datos.
110

Para el establecimiento de los valores en las categorías se tomó como base el UWES-S, con cinco categorías: "Muy bajo", "Bajo", "Promedio", "Alto" y "Muy alto". La Tabla No. 2 despliega los puntajes para el UWES-S (Schaufeli y Bakker, 2003).

Tabla 2. Atributos considerados y sus escalas de medida.

\begin{tabular}{|c|c|c|c|c|c|}
\hline \multirow[b]{2}{*}{ tegoría } & \multirow[t]{2}{*}{$\mathrm{Ca}$} & \multirow[t]{2}{*}{$\mathrm{Vi}$} & \multirow{2}{*}{$\begin{array}{l}\text { Dedic } \\
\text { ación }\end{array}$} & \multirow{2}{*}{$\begin{array}{r}\text { A } \\
\text { bsorción }\end{array}$} & \multirow{2}{*}{$\begin{array}{l}\text { Punta } \\
\text { je Total }\end{array}$} \\
\hline & & & & & \\
\hline & $\mathrm{M}$ & $<=$ & $<=1.3$ & $<=$ & $<=1.7$ \\
\hline uy bajo & & 2.0 & 3 & 1.77 & 7 \\
\hline & $\mathrm{Ba}$ & 2.0 & $1.34-$ & 1. & $1.78-$ \\
\hline jo & & $1-3.25$ & 2.90 & $78-2.33$ & 2.88 \\
\hline & $\operatorname{Pr}$ & 3.2 & $2.91-$ & 2. & $2.89-$ \\
\hline omedio & & $6-4.80$ & 4.70 & $34-4.20$ & 4.66 \\
\hline & $\mathrm{Al}$ & 4.8 & $4.71-$ & 4. & 4.67- \\
\hline to & & $1-5.65$ & 5.69 & $21-5.33$ & 5.50 \\
\hline & $\mathrm{M}$ & $>=$ & $>=5.7$ & $>=$ & $>=5.5$ \\
\hline uy alto & & 5.66 & 0 & 5.34 & 1 \\
\hline
\end{tabular}

\section{Resultados obtenidos de la agrupación}

Los grupos obtenidos a través de la técnica de clusterización usando el algoritmo a $\mathrm{K}$ medias se muestra en la Tabla 3. Para usar esta técnica se empleó herramienta de minería de datos Weka.

\section{Gr}


Periódico do Núcleo de Estudos e Pesquisas sobre Gênero e Direito Centro de Ciências Jurídicas - Universidade Federal da Paraíba V. 9 - $\mathrm{N}^{\mathrm{o}} 01$ - Ano 2020 - Spanish Edition ISSN | 2179-7137 | http://periodicos.ufpb.br/ojs2/index.php/ged/index

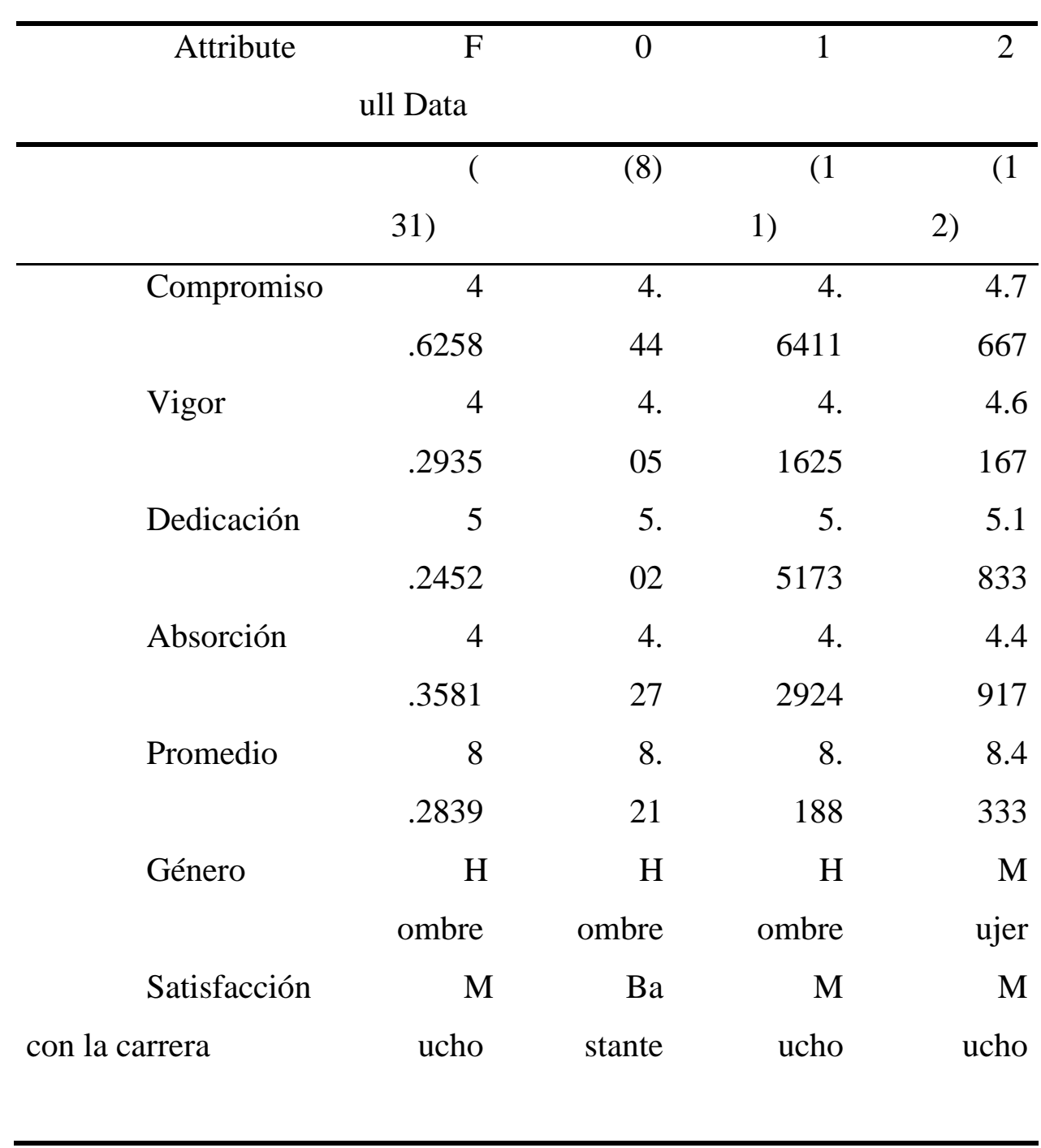

Tabla 3. Agrupación obtenida con el software Weka. 
En total se obtuvieron tres grupos, dos formados por hombres y uno por mujeres. En las agrupaciones se muestra que el grupo 0 se encuentra integrado por hombres con una puntuación de compromiso estudiantil de 4.44 definida como alta, en cuanto a las dimensiones que conforman el compromiso presentan valores de 4.05 (promedio), 5.02 (alto), 4.27(alto) en vigor, dedicación y absorción, respectivamente y un promedio de 8.21. Adicionalmente se les pregunto si se sentían satisfechos con la carrera, a lo cual respondieron que bastante.

El grupo 1 está formado por hombres, quienes presentan un alto compromiso estudiantil, con valores de vigor promedio con 4.1625, dedicación alta con 5.5173, absorción alta con 4.2924 , un promedio de calificaciones de 8.18, con una opinión de mucha satisfacción con su carrera.

El grupo 2 conformado por mujeres, quienes presentan un alto compromiso estudiantil, con valores de vigor promedio con 4.6167, dedicación alta con 5.1833, absorción alta con 4.4917, un promedio de calificaciones de 8.18, con una opinión de mucha satisfacción con su carrera.
Este grupo 0 en general se siente comprometido, medianamente vigoroso, altamente dedicado y absorto, es decir, para ellos tiene significado su carrera, tienen la voluntad de dedicar esfuerzo a su carrera. El grupo 1 esta comprometido con sus estudios, en mayor medida que el grupo 0, al igual que todas las variables que integran el compromiso estudiantil, y se corrobora al contestar a la pregunta con satisfecho en satisfacción con la carrera.

El promedio de su carrera es un poco menor en comparación que el grupo 0 . Este grupo se caracteriza por ser el mas dedicado, se sienten entusiasmados e inspirados por su carrera. El grupo $0 \mathrm{y}$ grupo 1 esta conformado por hombres a diferencia del grupo 2, que está integrado por mujeres, que presentan el más alto nivel de compromiso de la población, y superan en vigor y dedicación a los otros dos grupos, es decir, este grupo se siente satisfecho con sus estudios, con un estado positivo, inmerso en sus estudios, se encuentran orgullosas de sus estudios.

\section{Conclusiones}

En este estudio se llevó a cabo un análisis para conocer el compromiso estudiantil de los estudiantes de una 
universidad al sur de México. Se presentaron los estadísticos descriptivos que presentan coincidencias con la clusterización aplicada.

La clusterización es un proceso de minería de datos que permite agrupar dependiendo de las características presentadas. Para llevar a cabo este estudio se siguió el proceso de descubrimiento de conocimiento en base de datos (Knowledge Discovery in Databases, KDD).

En los resultados presentados se observó que el grupo 2 integrado por mujeres presentan un alto compromiso estudiantil, que se corrobora con una pregunta aplicada. Así mismo presentan un alto nivel de vigor y absorción, es decir presentan altos niveles de energía y concentración. El grupo 0 y 1 está formado por hombres, en el grupo 0 los estudiantes presentan un nivel de compromiso medio, el grupo 1 presenta un nivel de compromiso alto, así como un nivel de dedicación alto, incluso más alto que los otros dos grupos.

Con este tipo de estudios es posible conocer a la población estudiantil e identificar sus características, para realizar recomendaciones y partiendo de ello tomar decisiones en favor de los estudiantes universitarios.

Se propone continuar con este tipo de estudios, usando otras técnicas de minería de datos como el uso de árboles de decisión, correlaciones e incluso usar técnicas visuales que permitan realizar los análisis de una forma rápida y efectiva, mostrando de manera adecuada los datos multivariantes.

\section{Referencias}

Fayyad, U., Piatetsky-Shapiro, G., y Smyth, P. (1996). From data mining to knowledge discovery in databases. AI magazine, $\quad 17(3), \quad$ DOI http://dx.doi.org/10.1609/aimag.v17i3.1 $\underline{230}$

Gómez, C. M. V., y Gundín, O. A. (2016). Engagement, burnout y rendimiento académico en estudiantes universitarios y su relación con la prioridad en la elección de la carrera. Revista de Psicología y Educación, 11(1), 45-60.

Hernández, J., Ramírez, M.J., Ferri, C. (2004). Introducción a la Minería de Datos. España. Pearson 
Liébana-Presa, C., Fernández-Martínez, M., Vázquez-Casares, A. M., LópezAlonso, A. I., \& Rodríguez-Borrego, M. (2018). Burnout y engagement en estudiantes universitarios de enfermería. Enfermería Global, 17(50), 131-152.

Muñoz, M. A. C., y Morales, C. M. (2019). Nivel de engagement y su implicancia en el rendimiento académico en estudiantes universitarios de ciencias de la salud de la Unheval-Huánuco, 2015. Revista Boletín Redipe, 8(2), 131-139.

Pérez, María (2014). Minería de Datos a través de ejemplos. México. Alfaomega.

Schaufeli, W. y Bakker, A. (2003). UWES - Utrecht Work Engagement Scale Preliminary Manual. Revista Psicogente. Vol. 9. Núm. 1. pp. 6-9

Universidad Juárez Autónoma de Tabasco, (2005), Modelo Educativo. Villahermosa, Tabasco, México.

Weka (2019). Software de aprendizaje automático en Java. Recuperado de: https://www.cs.waikato.ac.nz/ml/weka/ 